\title{
Tertiary study on the application of augmented reality in education
}

\author{
Francisco Vinicius Nascimento da Silva, Pedro Henrique Araújo de Brito, \\ Ricardo Antônio Rebouças Celestino, Haniel Gomes Cavalcante, \\ Daniel Gleison Moreira Lira, Thaís de Almeida Barros, Mariela I. Cortés ${ }^{1}$ \\ ${ }^{1}$ Programa de Pós Graduação em Ciência da Computação - Universidade Estadual do Ceará \\ Avenida. Dr. Silas Munguba, 1700 - Itaperi, Fortaleza - CE, 60714-903 \\ ffrancisco.vinicius, pedrinho.brito, ricardo.celestino, \\ haniel.cavalcante, daniel.gleison, thais.almeida\}@aluno.uece.br, \\ mariela.cortes@uece.br
}

\begin{abstract}
The aim of this work is to carry out a tertiary study on the use of Augmented Reality (AR) in education, based on a set of five research questions. This review will greatly benefit researchers who wish to conduct research on the topic for the first time. In addition, this study extends to new researchers due to the compilation of important information that was collected through Systematic Literature Reviews - RSL. It was concluded that the research questions were answered based on the findings and it is also possible to observe the expansion of technologies, AR research fields and target audience by each author.
\end{abstract}

Resumo. O objetivo deste trabalho é a realização de um estudo terciário sobre o uso da Realidade Aumentada (RA) na educação, baseado num conjunto de cinco questões de pesquisa. Essa revisão beneficiará enormemente os pesquisadores que desejarem conduzir uma pesquisa sobre o tema pela primeira vez. Além disso, este estudo se estende a novos pesquisadores devido ao compilado de informações importantes que foram coletadas por meio de Revisões Sistemáticas da Literatura - RSL. Concluiu-se que as questões de pesquisa foram respondidas com base nas descobertas encontradas e também é possivel observar a expansão das tecnologias, campos de pesquisas da RA e publico alvo por parte de cada autor.

\section{Introduction}

Society has taken paths that have shaped the way we use knowledge, whether in the professional or educational sphere, forcing us, in a certain way, to adapt to the innovations brought by the advent of technologies in our daily lives.

In this scenario of globalization and reach of new knowledge, emerging technologies take a prominent place. For example, we can observe in recent years the increased use of augmented reality (AR), a technology that uses computer-generated graphic simulations overlaying reality to augment [Azuma et al. 2001]. AR is a generic term to refer to virtual elements that interact with the real environment. This technique has been widely used mainly due to the increase in compatible devices and the emergence of software that aims to facilitate the development of AR applications. Nowadays such applications can be created on personal computers of modest configurations and embedded in common devices. [Scavarelli et al. 2021] 
One of the areas that has been prominent in the use of augmented reality is education, applications range from early childhood education to higher education, as well as other non-formal educational spaces such as museums and libraries [Ibáñez and Delgado-Kloos 2018] [Garzón et al. 2019] [Pellas et al. 2019] [da Silva et al. 2019] [Ali et al. 2019]. Some systematic reviews have been done to map the state of the art of the use of augmented reality in educational contexts.

Therefore, the aim of this paper is to collect the secondary studies and conduct a tertiary study on the topic. Answering five research questions with varied themes of contributions, evolution, technologies, and what else is currently being used. To this end, sixteen systematic reviews from the ACM, IEEE, Springer, SciElo, MS Academic, and Scopus databases were analyzed.

In Section 1 of this paper, the theoretical framework necessary to understand the study is addressed, and in Section 2, works related to the study are presented. In Section 3 , the methodology used in the development of the article is shown. In Section 4, a discussion of the results is made, and in Section 5, the conclusion and future works are presented.

\section{Related Work}

Many researches are focused on the field of education. The discussion for the gaps that exist regarding the lack of real evidence that AR/VR are effective in education and also comparison of their use in learning spaces can be seen in [Scavarelli et al. 2021]. In [Queiroz et al. 2018a] maps AR/VR in education and how both can contribute to the identification of gaps, overlaps and opportunities in the area, as well as assist in pedagogical and public policy planning in Brazil. In [Ali et al. 2019] it is shown that few researches have occurred in real teaching environments and the difficulty of replicating mixed reality experiments in real educational spaces as well as the areas and subjects most frequently used with AR and mixed reality. Studies such as [da Silva et al. 2019] also propose a classification and discussion of studies that evaluate the educational aspects of AR systems and shows the evolution and type of research done from 2009 to 2017 in the area of education using AR, as well as which institutions are most involved in this type of study. Regarding the area of games for education, [Li et al. 2017a] classifies groups of learners, subjects, and learning environments to conclude that AR games for learning generally have positive effects.

\section{Methodology}

This review is shaped towards answering the following research questions:

- RQ1 - Is AR used in education? What is the contribution of using AR in education compared to the conventional methods?

Justification: This question aims to bring this research to life and is based on the reviews researched where a search and comparison between the learning methods used can be made.

- RQ2 - What types of AR technology are being used?

Justification: For this question, we will answer by citing the technologies used to execute AR techniques in education. 
- RQ3 - Which target audience has made use of AR in the learning process?

Justification: To answer this question it was observed which public made use of $\mathrm{AR}$ and their level of education.

- RQ4 - Which fields of education work with AR applications?

Justification: This research question was made so that we can explain which areas of education use AR techniques more frequently in their teaching methods.

- RQ5 - How has the use and research of AR evolved?

Justification: For this research question it is necessary to carry out a survey of future works proposed in the literature. Among the existing scientific databases SciELO, Microsoft Academic, ACM, IEEE, Scopus and Springer were chosen and the following strings were used in the search:

- augmented reality AND systematic review OR augmented reality AND literature review OR augmented reality AND literature analysis AND augmented reality AND review of studies.

As inclusion/exclusion criteria we evaluated the following factors:

- Be a systematic review;

- Comply with the structure protocols of a systematic review;

- Have the theme related to augmented reality in education;

- Results from the year 2015.

\subsection{Review development and protocol}

To conduct this systematic review we used the method developed by Ferenhof and Fernandes (2016), which uses database searches in order to allow the replication of research more easily, avoiding possible biases of researchers in choosing articles through exploratory methods, which is a disadvantage found in other methods of literature review, such as narrative review. The composition of this portfolio is carried out as follows:

Activity 1: Search strategy: The search strategy used the terms "augmented reality", "systematic review", "literature review", "literature analysis" and "review of studies". The intent of the search was to find results related to augmented reality so it used quotes searching the term "augmented reality". As the goal is also to search for systematic reviews, the terms "systematic review", "literature review", "literature analysis" and "review of studies" were used in quotes, as they all refer to reviews. The Boolean operators "AND" and "OR" were used to combine everything.

As inclusion and exclusion criteria, it was adopted:

- Be a systematic review;

- Comply with the structure protocols of a systematic review;

- Have the theme related to augmented reality in education;

- Results from the year 2015.

Activity 2: Searching databases: the databases chosen were: ACM, IEEE, Springer, SciElo, MS Academic and Scopus. The search string set up was: augmented reality AND systematic review OR literature review OR literature analysis OR review of studies. 
Activity 3: Standardized selection of articles: this phase comprised the reading of titles, abstracts and keywords of each article, and those that met the inclusion criteria were selected. After this filtering, sixteen articles remained.

Activity 4: Composing the research portfolio: after the selection made in activity 3 , the filtered articles were read in their entirety in order to extract the information that answered the research questions. The portfolio consists of 16 systematic reviews.

\section{Discussion of results}

The sixteen systematic reviews worked out for study, during its reading and evaluation process, terms were discovered that we used as a theme for each question. Each theme cites a data collection of pertinent information to answer the research questions.

The terms used to distinguish the themes of the questions were: Contribution, Technologies, Target Audience, Fields of Education and Evolution, where Contribution is related to the advantages that the reviews bring to the scientific community, Technologies is related to the types of materials and technologies used in the reviews we worked on, Target audience refers to the people who made use of the technologies, Fields of Education is where we have the target audience and the technologies applied and Evolution refers to the future work that each author left as a proposal in his review. Next we explain the answers to our research questions, where we separate each answer into its coded word.

\subsection{Augmented Reality Contributions}

In this section we show authors where we obtained the answers regarding the contributions that these reviews bring to the academic community, that is, answering the question 1, "What is the contribution of the use of AR in education in relation to the conventional methods used". As an answer to this question in our research we can highlight as main contributions a greater motivation on the part of students found in [Gómez García et al. 2020, LOPES et al. 2019a], [Raju and Joshith 2019, Queiroz et al. 2018b], facilities of augmented reality for handling materials and learning on the part of students were found in [Petrovich et al. 2018, Raju and Joshith 2019], augmented reality has also brought with it the implementation of new technologies for education that can be found in the works of [LOPES et al. 2019a, Gómez García et al. 2020], [Li et al. 2017b, Sirakaya and Alsancak Sirakaya 2020], [Petrovich et al. 2018, Queiroz et al. 2018b], [Raju and Joshith 2019]. Another contribution we can highlight is the mapping of pedagogical approaches found in the works of [Petrovich et al. 2018, Blattgerste et al. 2019], [Queiroz et al. 2018b] and the process of learning by interactive means facilitating the passage of information by users addressed in the works of [Li et al. 2017b, Sirakaya and Alsancak Sirakaya 2020], [Queiroz et al. 2018b, Raju and Joshith 2019].

\subsection{Technologies used}

In this section we have the answers from research question 2. The question was as follows, "What types of AR technology are being used?", for the answers, we assigned the term Technology to the authors who made use of Augmented Reality and Virtual Reality. The technologies we worked on to solve this research question were augmented reality glasses 
used in the interaction of students and pedagogues during course delivery, the papers that contain this content addressed were [Li et al. 2017a, Gómez García et al. 2020], [LOPES et al. 2019a, Sirakaya and Alsancak Sirakaya 2020], [Petrovich et al. 2018, Blattgerste et al. 2019], [Queiroz et al. 2018b, [Raju and Joshith 2019], [Scavarelli et al. 2021, Garzón et al. 2019], [Pellas et al. 2019, da Silva et al. 2019], [Ali et al. 2019, Ibáñez and Delgado-Kloos 2018, , Akçayır and Akçayır 2017, Li et al. 2017b], [Bacca-Acosta et al. 2014] and also the handling of teaching materials with AR technology whose function is to facilitate the handling of the content applied in classes in a more interactive way so that it motivates the student more during the learning process, found in [Li et al. 2017a, Gómez García et al. 2020], [LOPES et al. 2019a, Sirakaya and Alsancak Sirakaya 2020], [ [Petrovich et al. 2018, Blattgerste et al. 2019], [Queiroz et al. 2018b, Raju and Joshith 2019], [Scavarelli et al. 2021, Garzón et al. 2019], [Pellas et al. 2019, da Silva et al. 2019], [Ali et al. 2019, Ibáñez and Delgado-Kloos 2018], [Akçayır and Akçayır 2017, Li et al. 2017b], [Bacca-Acosta et al. 2014].

\subsection{Target Audience}

In this section we address the answers to question 3, "Which target audience made use of AR in the learning process?" To solve these questions we researched on the possible publics that each author was applying their studies, in this table we explain all the target publics that we found in our studies. The target audience that made use of Augmented Reality in education were students from the most diverse areas found in [Li et al. 2017a, Gómez García et al. 2020], [LOPES et al. 2019b, Sirakaya and Alsancak Sirakaya 2020], [Petrovich et al. 2018, Scavarelli et al. 2021], [Garzón et al. 2019, da Silva et al. 2019], [İbáñez and Delgado-Kloos 2018, Bacca-Acosta et al. 2014], [Li et al. 2017b. LOPES et al. 2019a], [Raju and Joshith 2019] and educators also from more diverse areas we find in [Scavarelli et al. 2021, Ibáñez and Delgado-Kloos 2018], [Garzón et al. 2019, Pellas et al. 2019], [Ali et al. 2019, Gómez García et al. 2020], [Queiroz et al. 2018b, Akçayır and Akçayır 2017]. There was also mention of developers focused on the application of this technology addressed in the works of [Pellas et al. 2019, Ali et al. 2019], [Queiroz et al. 2018b, Akçayır and Akçayır 2017].

\subsection{Fields of education}

In our studies, in each review read, we found besides the target audience, the fields of study that each audience belonged to, making it possible to elaborate the research question 4 "Which fields of education work AR applications?". Where we can see that the fields of education that work with augmented reality have been increasing as technology inserts itself into people's lives. They are Elementary/Middle Level: [Ali et al. 2019, Ibáñez and Delgado-Kloos 2018], [Li et al. 2017b, Gómez García et al. 2020], [LOPES et al. 2019a, Bacca-Acosta et al. 2014], [Sirakaya and Alsancak Sirakaya 2020, da Silva et al. 2019], [Pellas et al. 2019], [ Raju and Joshith 2019, Akçayır and Akçayır 2017], [Queiroz et al. 2018b], Engineering: [LOPES et al. 2019a, Sirakaya and Alsancak Sirakaya 2020], [IIbáñez and Delgado-Kloos 2018, Garzón et al. 2019], [Pellas et al. 2019. 
da Silva et al. 2019],

Ali et al. 2019,

Bacca-Acosta et al. 2014],

Sci-

ences in general:

[SIrakaya and Alsancak Sirakaya 2020,

Queiroz et al. 2018b], [Scavarelli et al. 2021, Garzón et al. 2019],

[Pellas et al. 2019, Ibáñez and Delgado-Kloos 2018], , Akçayır and Akçayır 2017, Bacca-Acosta et al. 2014], [Pellas et al. 2019, da Silva et al. 2019], [Bacca-Acosta et al. 2014], Mathematics: [Sirakaya and Alsancak Sirakaya 2020, Queiroz et al. 2018b], [Garzón et al. 2019, Pellas et al. 2019], [da Silva et al. 2019, Ibáñez and Delgado-Kloos 2018], , [Akçayır and Akçayır 2017], Architecture: [LOPES et al. 2019a, Queiroz et al. 2018b], [Garzón et al. 2019, Bacca-Acosta et al. 2014], Health: [LOPES et al. 2019a, Queiroz et al. 2018b], [Scavarelli et al. 2021, Garzón et al. 2019],

[da Silva et al. 2019,

Bacca-Acosta et al. 2014], Design: [LOPES et al. 2019a, Queiroz et al. 2018b], [Garzón et al. 2019, Bacca-Acosta et al. 2014], Nonformal learning spaces: [Scavarelli et al. 2021, Garzón et al. 2019], [Akçayır and Akçayır 2017, Bacca-Acosta et al. 2014] and Languages [Pellas et al. 2019].

\subsection{Evolution of works}

Our last question relates to future works and proposals left by each author. The answers of question 5 "What is the evolution of the use and research of AR?". As evolution of research authors who suggested to work on expanding research in the other fields of education were [Gómez García et al. 2020, LOPES et al. 2019a], [SIrakaya and Alsancak Sirakaya 2020],

[Blattgerste et al. 2019], [Akçayır and Akçayır 2017], authors who decided to pursue research with a focus on learning motivation were [Li et al. 2017b, LOPES et al. 2019a], [Gómez García et al. 2020], authors who observe the effectiveness of including AR in education and facilitating its use were [Scavarelli et al. 2021, Garzón et al. 2019], [Akçayır and Akçayır 2017, Bacca-Acosta et al. 2014], increase focus on exploring mixed reality virtual reality. Explore the use of AR with mixed reality were [Garzón et al. 2019, Pellas et al. 2019], [Petrovich et al. 2018, Blattgerste et al. 2019], Explore use of other media, include new evaluative measures were [İbáñez and Delgado-Kloos 2018] and finally include education professionals in the development process and take into account the complexity of human behavior are in the works of [da Silva et al. 2019].

\section{Conclusions and future work}

RA is an area of knowledge that provides several opportunities for scientific research and innovation, as it is still considered a constantly growing technology, and also because it offers users better interactions with the virtual world, through intuitive interfaces [Nakamoto et al. 2012]. In Table 1 we can find the main findings with the answers to our research questions. We also conclude that the questions proposed in this tertiary study were answered based on the analysis of the systematic reviews worked on. During our research we can observe that augmented reality is something that is more present in several areas of education. The audience is somewhat broad and has been expanding as this technology evolves. As we have shown, the fields of education that augmented reality works are very diverse, this growth is proportional to the growth of the target audience that uses this technology. And we can also observe that each author always aims to expand their 
research, whether through the public, areas of education and even expanding the fields of research in which it is possible to work with augmented reality.

Table 1. Findings of Research Questions

\begin{tabular}{|l|l|}
\hline \multicolumn{2}{|c|}{ Question } \\
\hline $\begin{array}{l}|c| \\
\text { What is the contribution of the } \\
\text { in relation to the } \\
\text { conventional methods used? }\end{array}$ & $\begin{array}{l}\text { Promote improvements in the sharing of knowledge } \\
\text { sharing among students and increase the } \\
\text { motivation of the contents taught. }\end{array}$ \\
\hline $\begin{array}{l}\text { What types of AR technologies are being } \\
\text { used? }\end{array}$ & $\begin{array}{l}\text { VR and AR Glasses; } \\
\text { Diactic materials in AR; } \\
\text { AR Applications. }\end{array}$ \\
\hline $\begin{array}{l}\text { Which target audience made } \\
\text { use of AR in the } \\
\text { learning process? }\end{array}$ & $\begin{array}{l}\text { Elementary, Middle } \\
\text { and Higher Students. }\end{array}$ \\
\hline $\begin{array}{l}\text { Which edu- } \\
\text { cation fields found work } \\
\text { with } \\
\text { AR applications? }\end{array}$ & $\begin{array}{l}\text { Health; Sciences; Math; } \\
\text { Pedagogy; Architecture. }\end{array}$ \\
\hline $\begin{array}{l}\text { What is the evolution of } \\
\text { use and research } \\
\text { on AR? }\end{array}$ & $\begin{array}{l}\text { Explore the use of other media; } \\
\text { Include new evaluative measures. }\end{array}$ \\
\hline
\end{tabular}

\section{References}

Akçayır, M. and Akçayır, G. (2017). Advantages and challenges associated with augmented reality for education: A systematic review of the literature. Educational Research Review, 20:1-11.

Ali, A. A., Dafoulas, G. A., and Augusto, J. C. (2019). Collaborative educational environments incorporating mixed reality technologies: A systematic mapping study. IEEE Transactions on Learning Technologies, 12(3):321-332.

Azuma, R., Baillot, Y., Behringer, R., Feiner, S., Julier, S., and Macintyre, B. (2001). Recent advances in augmented reality. ieee comput graphics appl. Computer Graphics and Applications, IEEE, 21:34 - 47.

Bacca-Acosta, J., Baldiris, S., Fabregat, R., Graf, S., and Kinshuk, D. (2014). Augmented reality trends in education: A systematic review of research and applications. Educational Technology and Society, 17:133-149.

Blattgerste, J., Renner, P., and Pfeiffer, T. (2019). Augmented reality action assistance and learning for cognitively impaired people: a systematic literature review. In Proceedings of the 12th ACM International Conference on PErvasive Technologies Related to Assistive Environments, pages 270-279.

da Silva, M. M. O., Teixeira, J. M. X. N., Cavalcante, P. S., and Teichrieb, V. (2019). Perspectives on how to evaluate augmented reality technology tools for education: a systematic review. Journal of the Brazilian Computer Society, 25(1):3. 
Garzón, J., Pavón, J., and Baldiris, S. (2019). Systematic review and meta-analysis of augmented reality in educational settings. Virtual Reality, 23(4):447-459.

Gómez García, G., Rodríguez Jiménez, C., and Marín Marín, J. A. (2020). La trascendencia de la realidad aumentada en la motivación estudiantil. una revisión sistemática y meta-análisis. ALTERIDAD. Revista de Educación, 15(1):36-46.

Ibáñez, M.-B. and Delgado-Kloos, C. (2018). Augmented reality for stem learning: A systematic review. Computers \& Education, 123:109-123.

Li, J., van der Spek, E. D., Feijs, L., Wang, F., and Hu, J. (2017a). Augmented reality games for learning: A literature review. In Streitz, N. and Markopoulos, P., editors, Distributed, Ambient and Pervasive Interactions, pages 612-626, Cham. Springer International Publishing.

Li, J., Van der Spek, E. D., Feijs, L., Wang, F., and Hu, J. (2017b). Augmented reality games for learning: A literature review. In International Conference on Distributed, Ambient, and Pervasive Interactions, pages 612-626. Springer.

LOPES, L. M. D., VIDOTTO, K. N. S., POZZEBON, E., and FERENHOF, H. A. (2019a). Inovações educacionais com o uso da realidade aumentada: uma revisão sistemática. Educação em Revista, 35.

LOPES, L. M. D., VIDOTTO, K. N. S., POZZEBON, E., and FERENHOF, H. A. (2019b). Inovações educacionais com o uso da realidade aumentada: Uma revisão sistemática. Educação em Revista, 35.

Nakamoto, P. T., Carrijo, G. A., Cardoso, A., Lima, L. V. d. O., and Lopes, E. J. (2012). Estratégia de engenharia de requisitos para ambientes de realidade aumentada. JISTEM - Journal of Information Systems and Technology Management, 9:607-626.

Pellas, N., Fotaris, P., Kazanidis, I., and Wells, D. (2019). Augmenting the learning experience in primary and secondary school education: a systematic review of recent trends in augmented reality game-based learning. Virtual Reality, 23(4):329-346.

Petrovich, M., Shah, M., and Foster, A. (2018). Augmented reality experiences in informal education. In 2018 IEEE International Conference on Teaching, Assessment, and Learning for Engineering (TALE), pages 815-819. IEEE.

Queiroz, A. C. M., Tori, R., Nascimento, A. M., and d. S. Leme, M. I. (2018a). Augmented and virtual reality in education: The role of brazilian research groups. In 2018 20th Symposium on Virtual and Augmented Reality (SVR), 2018 20th Symposium on Virtual and Augmented Reality (SVR), pages 170-175.

Queiroz, A. C. M., Tori, R., Nascimento, A. M., and Leme, M. I. d. S. (2018b). Augmented and virtual reality in education: The role of brazilian research groups. In 2018 20th Symposium on Virtual and Augmented Reality (SVR), pages 170-175. IEEE.

Raju, N. and Joshith, V. (2019). Augmented reality in english language pedagogy: An innovative techno culture for contemporary classrooms-a meta review.

Scavarelli, A., Arya, A., and Teather, R. J. (2021). Virtual reality and augmented reality in social learning spaces: a literature review. Virtual Reality, 25(1):257-277.

Sirakaya, M. and Alsancak Sirakaya, D. (2020). Augmented reality in stem education: A systematic review. Interactive Learning Environments, pages 1-14. 